\title{
Textile Tragmittel in der Fördertechnik
}

Dipl.-Ing. Thorsten Heinze, Markus Speck, M.Sc.

Technische Universität Chemnitz

Institut für Fördertechnik und Kunststoffe

Professur Fördertechnik

\section{Motivation}

\subsection{Erhöhung der Förderkapazitäten- und längen}

In der Fördertechnik spielen Tragmittel wie Seile, Riemen und Ketten als Übertragungs- und Verbindungselemente eine wesentliche Rolle. Dabei sind Seile für den technischen Einsatz vornämlich Drahtseile, mit Hauptanwendung in sicherheitsrelevanten Bereichen wie Aufzügen, Kränen und Schachtförderanlagen [Ver04]. Diese Präferenz liegt in dem Vorteil begründet, dass durch parallel angeordnete lasttragende Elemente ein redundanter Aufbau realisiert ist, der ein abruptes Versagen, wie bei den Kettentrieben verhindert. Durch regelmäßige Inspektionen können so kritische Zustände erkannt und Seile rechtzeitig ausgetauscht werden. Darüber hinaus macht seine eingehende Erforschung und stetige Weiterentwicklung das Drahtseil zu dem Tragmittel mit der höchsten Betriebssicherheit.

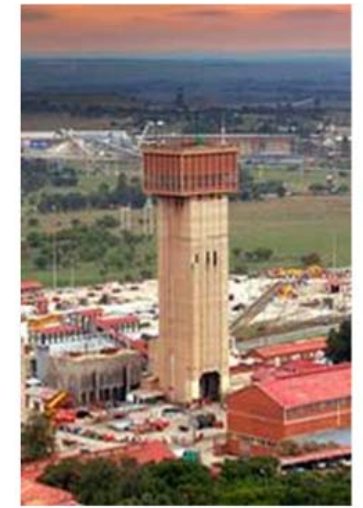

Goldmine TauTona $(3600 \mathrm{~m})$

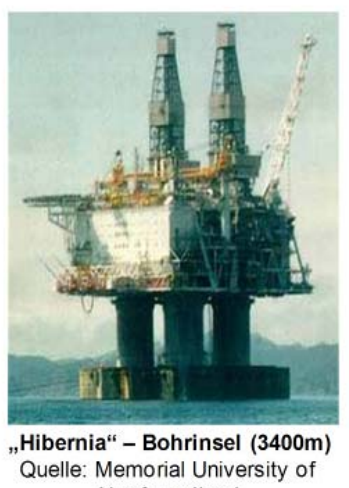

Newfoundland

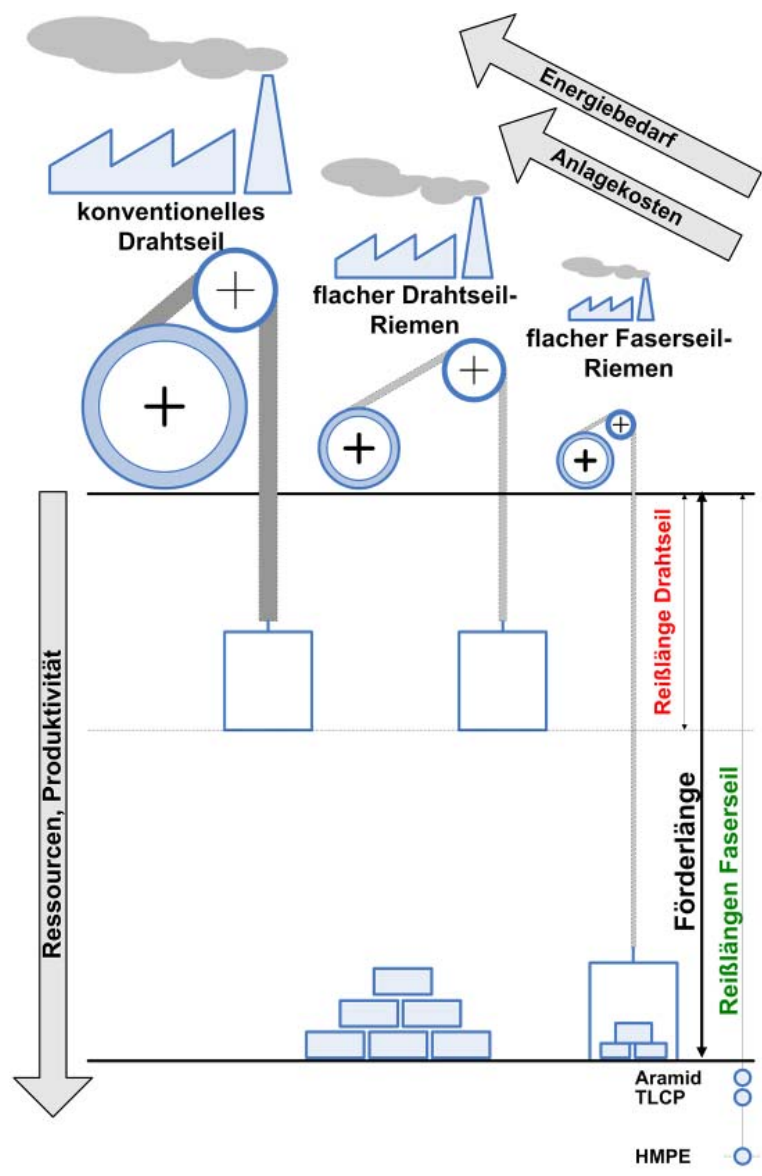

Abbildung 1: Technische Grenzen und Wirtschaftlichkeit von Aufzügen 
Dennoch werden durch die stetig wachsenden Anforderungen an heutige Drahtseile technische Grenzen u.a. durch die eingeschränkte Biegeflexibilität und die hohe Eigenmasse berührt, aus denen sich neue Erfordernisse hinsichtlich der Werkstoffe und Seilkonstruktionen ergeben. So dokumentieren Gebäude, wie das Burj Khalifa [Bla08], [Oti10] in Dubai mit einer Gesamthöhe von 828m einen Fortschritt, denen die konventionellen Drahtseile nicht Schritt halten. Ihre maximalen Förderhöhen liegen in der Personenaufzugstechnik, begrenzt durch Seilgewicht, Sicherheiten und Laufzeit bei ca. 500m. Dies erfordert mehrere übereinander angeordnete Aufzüge, wodurch höhere Analgekosten, ein höherer Zeitaufwand und logistische Probleme bei der Benutzung in Kauf genommen werden müssen.

Neben diesen populären Beispielen gibt es zahlreiche weitere Anwendungen, wie Schachtanlagen in Minen und Bergwerken mit Fördertiefen von über 3500m. Durch kleinere Sicherheiten einerseits und kleinere Förderströme andererseits, können Förderlängen bis zu 3000m [Sie04] realisiert werden. Gebräuchlicher sind jedoch auch hier Reihenschaltungen kleinerer Seiltriebe mit Förderhöhen unter 1000m, da größere Drahtseil-Förderanlagen im Allgemeinen als unwirtschaftlich angesehen werden.

Generell gilt, das beim Lauf über Scheiben der Seilquerschnitt hinreichend groß sein muss, um die geforderte Stranglast sicher zu übertragen, andererseits klein genug, um die lokalen Druck- und Zugspannungen sowie die Relativbewegungen klein zu halten. Durch eine Parallelanordnung von Seilen kann die Förderlast auf mehrere Zugstränge verteilt, die effektive Tragmittelhöhe reduziert und damit die Lebensdauer erhöht werden. Auf diese Weise konnte die Effizienz der Drahtseile nochmals gesteigert, die Forderung nach größeren Förderlängen jedoch nur unwesentlich bedient und die technischen Grenzen nicht durchbrochen werden. Dies ist grundsätzlich nur zu erreichen, indem die Zugfestigkeit des Aufzugsseils weiter gesteigert und/oder dessen Gewicht deutlich reduziert wird. Diese Optimierungsmöglichkeiten sind bei den Stahldrahtseilen bereits ausgeschöpft, da ihre Zugfestigkeit praktisch nicht weiter gesteigert und ihre Eigenmasse nicht verringert werden kann.

Im Gegensatz dazu bedienen moderne Faserseile beide Forderungen. Sie besitzen gegenüber Drahtseilen eine 1,5-3fach höhere Zugfestigkeit und ein 7-8fach geringeres Tragmittelgewicht, wodurch die Reißlängen [Hea08] 8-14mal höher sind und damit größere Förderhöhen bzw. ein energieeffizienterer Materialfluss realisiert werden kann. Dies bedeutet, dass Reihenschaltungen von Aufzügen entfallen und/oder wesentlich höhere Förderkapazitäten, eine längere Gebrauchsdauer der Seile, längere Wartungsintervalle und geringere Ausfallzeiten erreicht werden. Das Ziel einer höheren Wirtschaftlichkeit kann aber auch dadurch verfolgt werden, das bei gleicher Lebensdauer und Nutzlast die Treibscheibendurchmesser und mit innen die Antriebsmomente, Antriebe sowie die Investitions- und Betriebskosten verringert werden.

Moderne Faserseile bestehen aus hochfesten, hochmodularen Polymerfasern, sog. HM-HT-Fasern (high modulus - high tenacity), die im Unterschied zu den 
konventionellen Synthesefasern durch lineare, gleichsinnig orientierte Molekülketten sehr hohe axiale Steifigkeiten und Festigkeiten [Hea08],[Mor08] erreichen.

$\mathrm{Zu}$ den wesentlichen Vorteilen der HM-HT-Faserseile gegenüber Drahtseilen gehören:

- die Erweiterung der technischen Machbarkeit durch Förderlängen oberhalb der Reißlänge von Drahtseilen,

- die Verbesserung der Energiebilanz durch geringere bewegte Massen, Antriebsleistungen und Betriebskosten,

- die Verbesserung der Betriebssicherheit durch höhere Sicherheitsfaktoren und Gebrauchsdauer,

- die Erhöhung der Nutzlasten durch geringeres Seilgewicht,

- die Erhöhung des Komforts durch Wegfall von Umsteige- bzw. Umladestationen.

Die HM-HT-Fasern müssen jedoch neben dem geringen Gewicht und der hohen Zugfestigkeit eine Reihe weiterer Eigenschaftsmerkmale, wie sie in (Abbildung 2) angezeigt sind, vollständig oder durch Synergie in hybriden Werkstoffen erfüllen, bevor sie in Tragmitteltrieben einsetzbar sind. Das Netzdiagramm zeigt in einem Benchmarking gegenüber dem Referenzwerkstoff Stahl die Stärken und Schwächen möglicher Substituenten. So muss insbesondere das anisotrope Materialverhalten der Kunststoffe besseren Eingang in die Seildimensionierung finden. Die geringe Druck- und Querfestigkeit in Verbindung mit der fehlenden Formstabilität sowie daraus resultierender Faserknickung und diffusen Faserverschiebungen tragen entscheidend zu einer Verminderung der Lebensdauer laufender Faserseile bei.

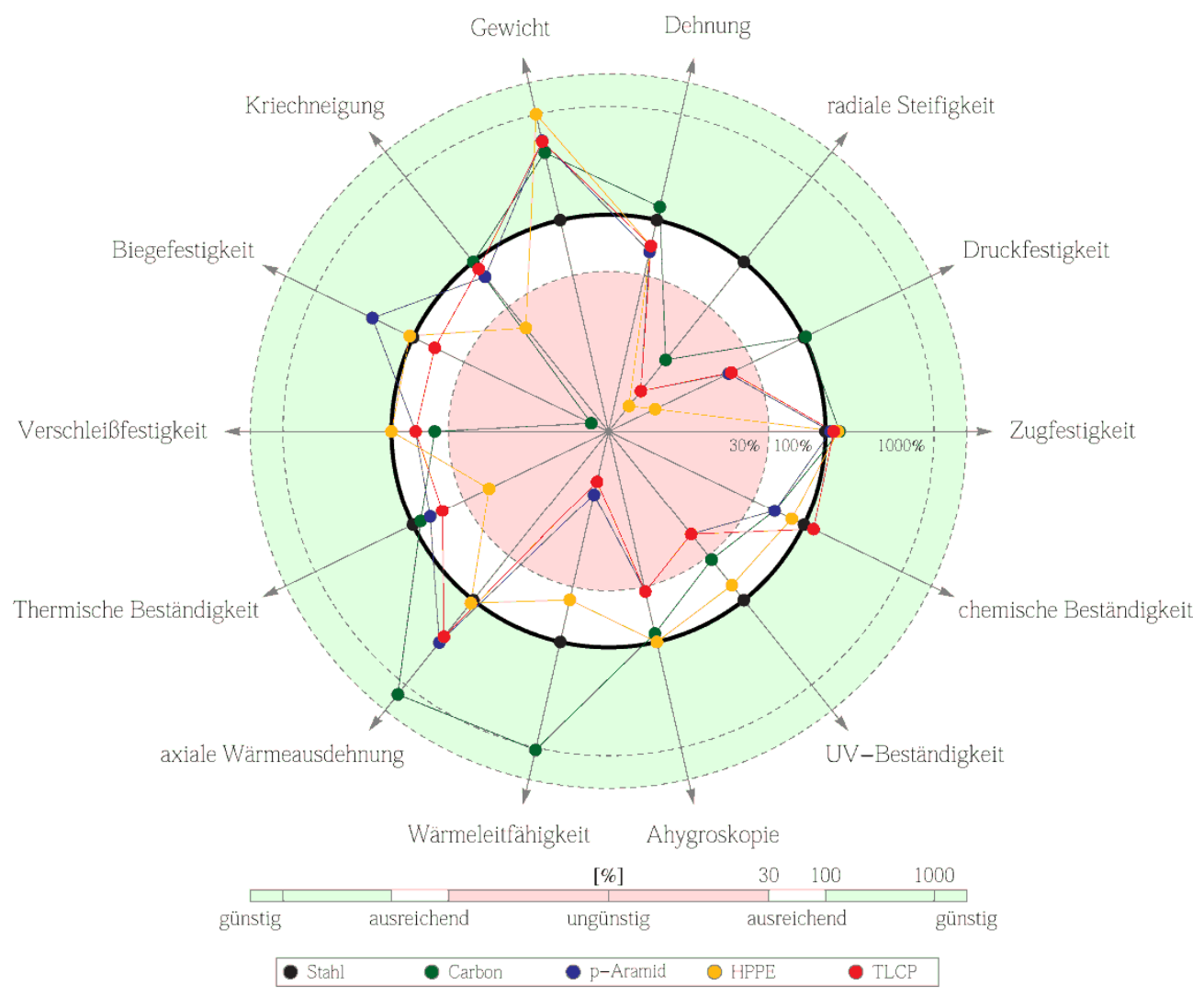

Abbildung 2: Vergleich von Materialien für den Einsatz in Tragmitteln 


\subsection{Tragmittelkonstruktion}

Zur Herstellung laufender, d.h. auf Wechselbiegung belasteter, Seile stehen grundsätzlich die Technologien Verdrehen und Flechten zur Verfügung. Zugfeste Drahtseile können aufgrund der Biegespannungen nur gedreht (geschlagen) hergestellt werden. Gedrehte Seilkonstruktionen haben, wie der Übersicht in Abbildung $3 \mathrm{zu}$ entnehmen ist, keine Überkreuzungsstellen wie Seilgeflechte, wodurch die Lebensdauer gedrehter Drahtseile gegenüber geflochtenen Drahtseilen größer ist.

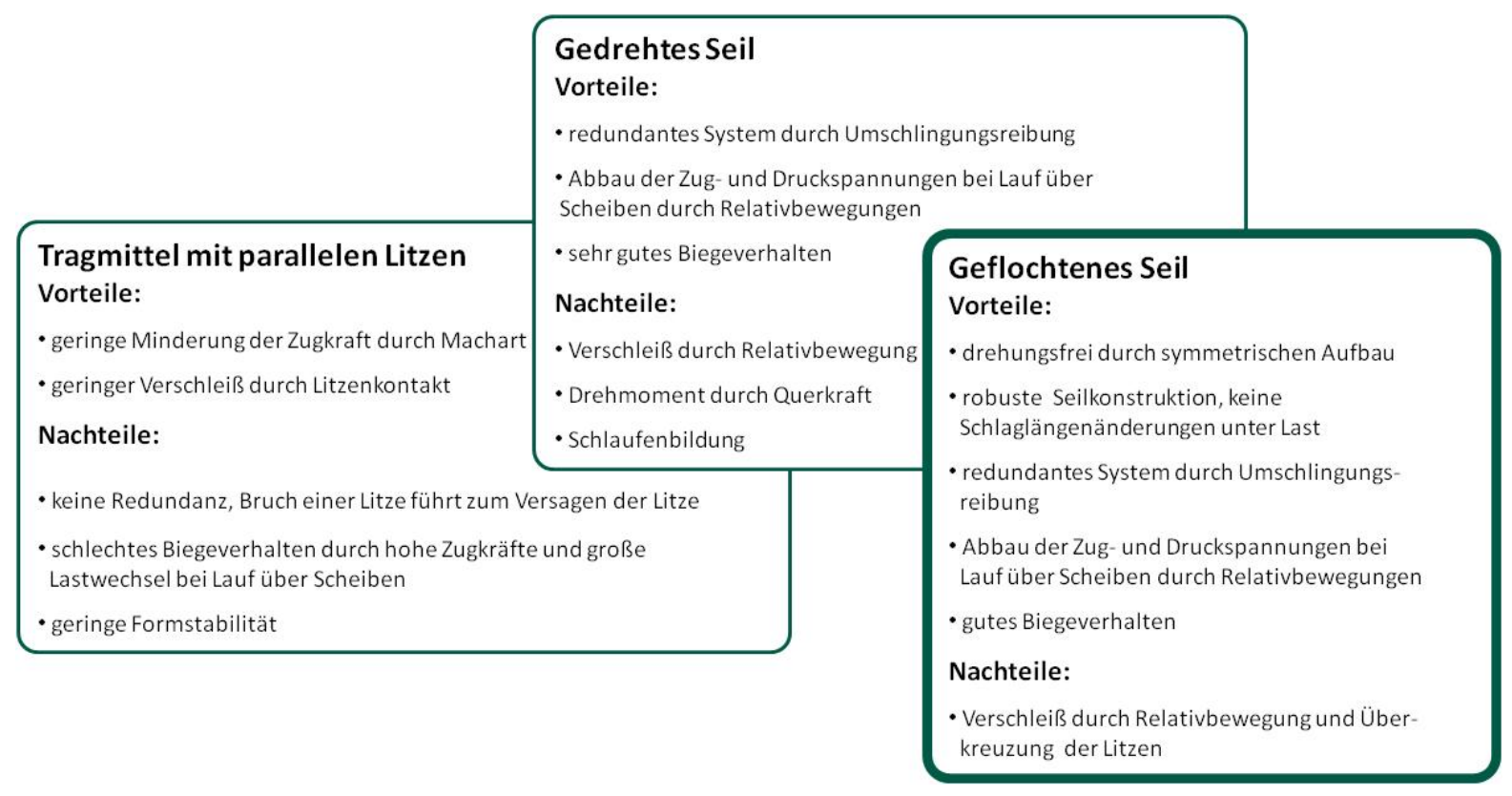

Abbildung 3: Vergleich verschiedener Tragmittel-Macharten

Die Hauptnachteile geschlagener Seile sind zum einen die Neigung zum Auf- bzw. Zudrehen des Seilverbandes bei Schrägzug oder anderen während der Handhabung bzw. Montage initiierten Zwangsverdrehungen und zum Anderen der prinzipbedingte mit einer Schlaglängenänderung einhergehende Momentenausgleich [Ver04] des Seils unter Last, welcher insbesondere bei großen Fördergewichten und -strecken zum Tragen kommt. Hierbei dreht sich das Seil infolge des hohen Eigengewichtes in Richtung des größeren Momentes auf. Da das Seil gegen Verdrehen gesichert ist, bleibt die Anzahl der Verdrehungen gleich, jedoch wird die Schlaglänge nach obenhin größer und nach unten kleiner [Ver05]. Diese verschiedenen Ursachen für Längendifferenzen führen zu einer Schädigung des Tragmittels, die hauptverantwortlich dafür sind, dass z.B. Schachtförderseile nie die, den Auslegungsvorschriften zugrunde gelegte, Lebensdauer erreichen.

Geflochtene Seile sind im Gegensatz zu den gedrehten Seilen nahezu drehungsfrei. Durch gegenläufige, sich überkreuzende Litzen entsteht ein symmetrischer Seilaufbau, der die wirkenden Drehmomente der jeweils paarweise agierenden Litzen kompensiert. Da Kunststofffilamente anders als Stahldrähte biegeschlaffe Gebilde sind, können die Krümmungsradien sehr klein gewählt werden, ohne dass im Material Zwangsspannungen auftreten. Die Firma DSM, ein Hersteller hochfester 
Fasern, hat in diesem Zusammenhang eine Studie zur Biegewechselfestigkeit von Dyneema-Seilen in Auftrag gegeben [Vog98], die keine signifikanten Unterschiede zwischen der Lebensdauer geflochtener und gedrehter Faserseile nachweisen konnte.

Des Weiteren werden durch die Überkreuzungen der Litzen geflochtener Seile, die Fasern abschnittsweise geklemmt, wodurch keine Schlaglängenänderungen, wie bei den gedrehten Seilen auftreten. Diese Umstände legen den Schluss nahe, dass geflochtene Seile besonders bei sehr großen Förderlängen eingesetzt werden können. Das Seilgeflecht ist somit ein Alleinstellungsmerkmal textiler Tragmittel, das einerseits durch Überkreuzungsstellen festigkeitsmindernd wirkt, jedoch andererseits Drehungsfreiheit und einen stabileren Seilverbund als gedrehte Seile bietet.

\section{Beschichtung von Seilen}

Eine Vielzahl von Veröffentlichungen hat gezeigt, dass eine Schmierung bzw. Beschichtung der HM-HT-Fasern oder Litzen, mit dem Ziel die innere Reibung zu vermindern, die Lebensdauer bei Wechselbiegung von HM-HT-Faserseilen erhöht. Dabei reduziert die Beschichtung nicht nur den Faserabrieb, sondern auch die mit der Erwärmung einhergehende Festigkeitsminderung und Kriechverformung. Als ein weiterer dominanter Schädigungsmechanismus ist die Druck- bzw. Knickempfindlichkeit der, auf höchste Zugfestigkeit, gezüchteten Fasern und die fehlende Formstabilität der aus innen hergestellten Seile zu sehen. So kann die Lebensdauer wesentlich gesteigert werden, wenn es gelingt, die Fasern am Ausknicken zu hindern und die Verschiebung der Zug-/Druckzonen sowie die Walkarbeit durch einen verformungsarmen Tragmittelquerschnitt zu vermindern. In Abbildung 4 sind die Auflageverhältnisse verschiedener Seilkonstruktionen dargestellt.

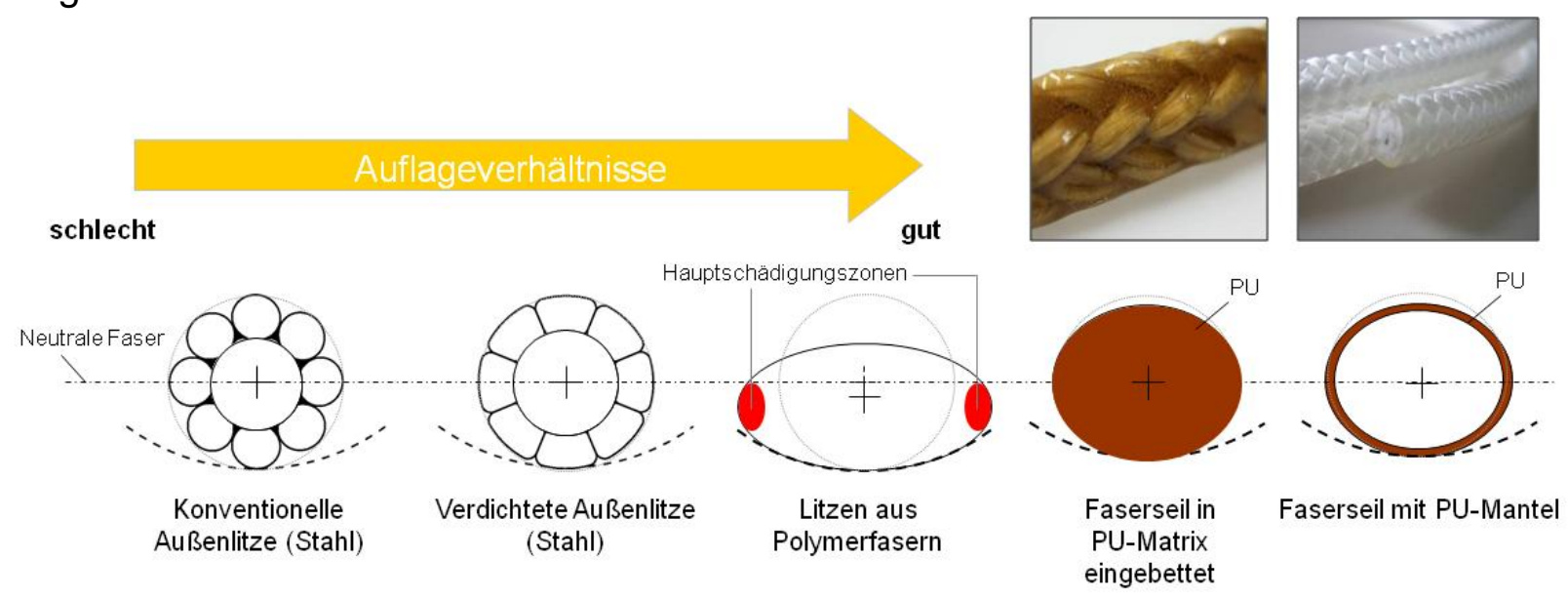

Abbildung 4: Auflageverhältnisse zwischen Seil und Seilscheibe 


\subsection{Seilimprägnierung}

Einen Ansatz bietet die Einbettung des gesamten Seiles in eine PU-Matrix. Der Matrixwerkstoff bewirkt dabei eine Bauteildämpfung, eine Reduzierung der lokalen Flächenpressungen an den Seilgeflechten, eine Verbesserung der Treibfähigkeit durch höhere Reibwerte und eine Verschleißminderung durch die Zwangsordnung der Fasern. Darüber hinaus werden die Relativbewegungen behindert und lediglich eine geführte Verformung, durch den stärker dehnenden Matrixwerkstoff, zugelassen. Mittels Linien-Lasersensor konnte, wie in Abbildung 5 dargestellt, eine verminderte Querschnittsdeformation in der Rille nachgewiesen werden. Die Seile sind hierzu kraftgeregelt mit einer Vorkraft von jeweils $3 \mathrm{kN}$ belastet wurden. Das Querschnittsprofil von Seil und Rille wurde überlagert und aus der Querschnittsfläche ein Referenzkreis gebildet, auf den sich die Konturabweichung bezieht. Bei $180^{\circ}$ wurde der Rillengrund definiert.
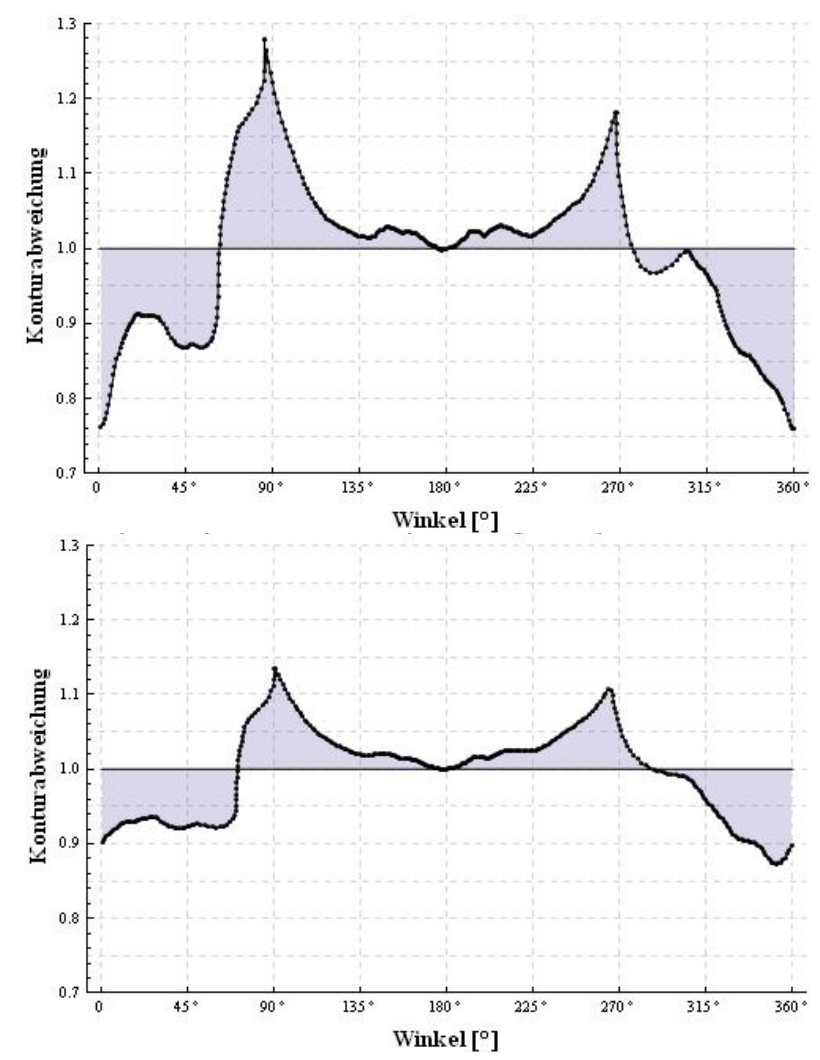
a) unbeschichtetes Seil
b) beschichtetes Seil

Abbildung 5: Querschnittsdeformation 6mm-Technora-Seil

Für den Funktionsnachweis wurde ein para-aromatisches Copolyamid (Technora) in einer 6mm-Rundgeflechtkonstruktion eingesetzt. Nach Entfernen der Faserschlichte durch eine kombinierte Behandlung mit Seifenlauge und Ultraschall wurde die Struktur bei $40^{\circ} \mathrm{C}$ getrocknet und anschließend an einer Beschichtungsanlage im Tauchverfahren mit einer Polyurethan-Dispersion vollkommen durchdrängt und das Wasser im anschließenden Trockenprozess ausgeschwitzt. 


\subsection{Mantelextrusion}

Eine weitere Möglichkeit der Beschichtung ist die Mantelextrusion, bei der eine ca. $1 \mathrm{~mm}$ dicke PU-Schicht auf das Seil aufgetragen wird. Beim Beschichten von Kunststoffen mittels Extrusion, überlagern sich die mechanische Verklammerung und die Adsorption. Zunächst wird die Faserschlichte auf oben beschriebene Weise entfernt und im Weiteren die Struktur vollständig oder an der Oberfläche mit einem als Zwischenschicht genutzten Haftvermittler imprägniert. Dieser wird z.B. in Form einer Polyurethan-Dispersion aufgebracht und der Wasseranteil anschließend ausgetrocknet. Der Haftvermittler ist aus zweierlei Gründen notwendig. Einerseits wirkt durch die relativ glatte Extrusionsschicht lediglich eine makroskopische (durch die Flechtstruktur des Seiles) nicht aber eine mikroskopische Verklammerung und andererseits ist die adhäsive Wirkung zwischen thermoplastischen Polyurethanen, die zum Extrudieren genutzt werden, und den meisten Faserwerkstoffen nicht optimal. Die Haftung zum Seilwerkstoff einerseits, als auch zum Extrusionsmaterial andererseits ist aufgrund der chemischen Ähnlichkeit gut. Sie kann sich sogar durch Diffusions- und eventuell auftretende Reaktionsprozesse im Grenzflächenbereich verstärken. Abschließend kann der Extrusionsvorgang mit einem thermoplastischen Werkstoff durchgeführt werden, dessen Verarbeitungstemperatur auf die Eigenschaften des Seilwerkstoffes abgestimmt ist.

\section{$3 \quad$ Ergebnisse zur Seilimprägnierung}

\subsection{Abrasionstest}

Ein Schädigungsmechanismus textiler Tragmittel ist der Oberflächenverschleiß, der u.a. durch Mikroschlupf an Treib- und Umlenkscheiben entstehen kann. An einem Abrasionsprüfstand für Seile wurde der Einfluss der Seilimprägnierung auf den Oberflächenverschleiß untersucht. Ein Pendel mit prismatischem Reibkörper drückt dabei auf das vorgespannte Prüfseil und vollführt eine Hubbewegung. Der Test wurde bei einer Vorkraft von $100 \mathrm{~N}$ und 25 Doppelhüben/min durchgeführt. Das unbeschichtete Seil erreichte, wie in Abbildung 6 zu sehen, 95.000 Doppelhübe. Dem gegenüber wies das beschichtete Seil nach 500.000 Doppelhüben einen Oberflächenabtrag von ca. 15\% auf, war jedoch durch den vollständig in das Seil eingedrungen Matrixwerkstoff noch immer nicht zerstört.

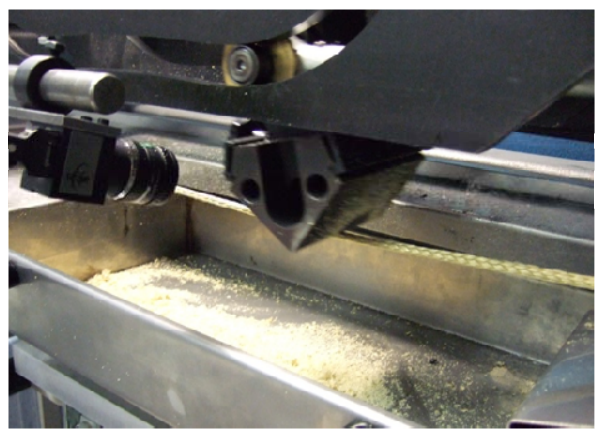

Abrasionstest mit 100N Vorkraft. 25 Hübe/min

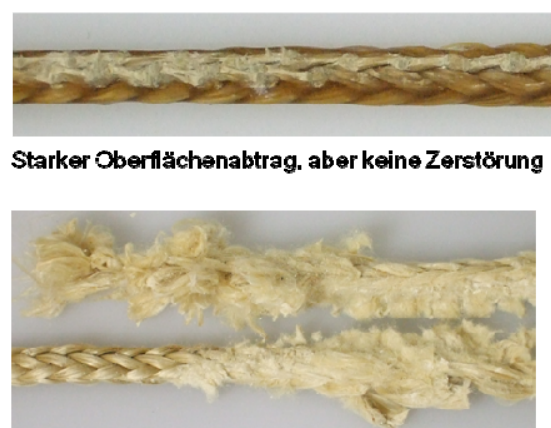

Komplette Zerfaserung des unbeschichteten Seils

\subsection{0 Doppelhübe}

\author{
95.000 \\ Doppelhübe
}

Abbildung 6: Abrasionstest 


\subsection{Treibfähigkeit}

Treibscheibenantriebe finden größtenteils Anwendung in Aufzügen und Schachtförderanlagen. Um einen sicheren Betrieb zu gewährleisten, muss die Treibfähigkeit, d.h. der Reibschluss zwischen Tragmittel und Treibscheibe ausreichend groß sein. An einem Treibscheibenprüfstand wurde der Einfluss der Seilbeschichtung auf die Treibfähigkeit (Abbildung 7) untersucht.

Der Prüfstand basiert auf dem eytelweinschen Modell der Umschlingungsreibung, bei dem sich der Reibwert aus dem Verhältnis der Kräfte und dem Umschlingungswinkel ermitteln lässt. Für die Versuche wurde eine Prüfscheibe mit Standard-Rundrille $(r=0,53 d)$ verwendet. Die Prüfkraft wurde auf $2 \mathrm{kN}(5 \% \mathrm{MBL})$ in der Start-StopBetriebsart eingestellt. Während das unbeschichtete Seil nach 100 Start-StopVersuchen einen relativ niedrigen Haftreibwert aufwies, war der Reibwert des beschichteten Seiles ca. $5 \mathrm{mal}$ so groß.

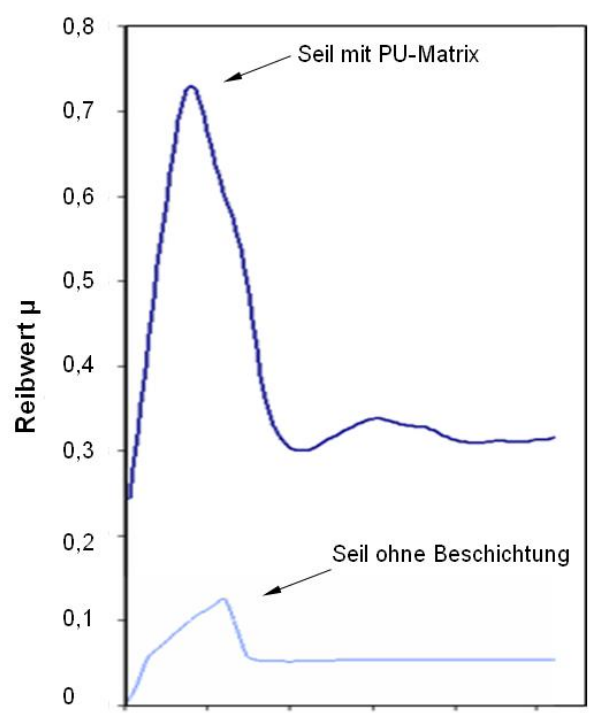

Abbildung 7: Reibwertverläufe im Start-Stop-Versuch

\subsection{Windentest}

Der Einsatz von Seilwinden ist bei vielen Anwendungen wie in Krananlagen, Schleppwinden etc. angezeigt. Insbesondere die Mehrlagenwickelung führt zu einer starken aus mehreren Belastungsparametern überlagerten Beanspruchung. In einem Windentest mit drei Seillagen, einem Prüfgewicht von $70 \mathrm{~kg}$ und einer Prüfgeschwindigkeit von $50 \mathrm{~m} / \mathrm{min}$ wurden für das beschichtete und unbeschichtete Seil jeweils 5000-Wickelzyklen gefahren. Abbildung 8 zeigt eine optisch wesentlich stärkere Schädigung des unbeschichteten Seiles. Anschließende Zugversuche bestätigten diesen ersten subjektiven Eindruck. Das unbeschichtete Seil wies eine Restbruchkraft von $45 \%$, das imprägnierte Seil von über $80 \%$ auf. 


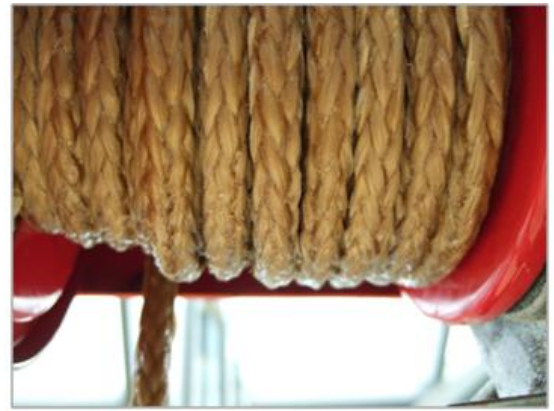

Technora-Seil, PU-Beschichtung

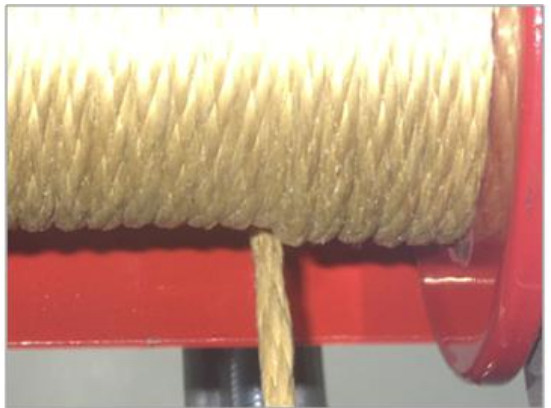

Technora-Seil, ohne PU-Beschichtung

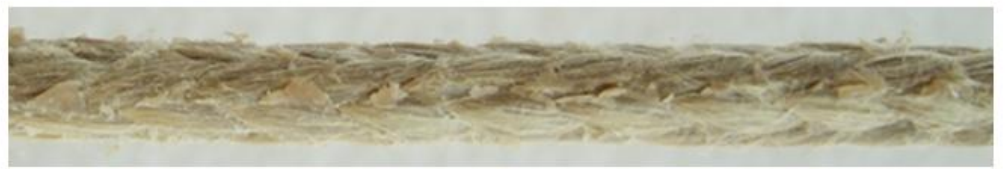

5.000-Wickelzyklen, Seil mit PU-Beschichtung

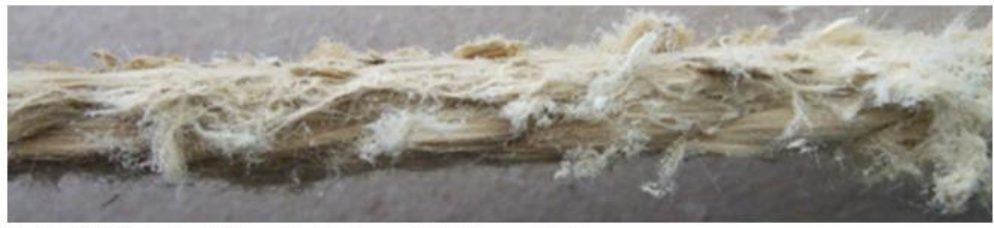

5.000-Wickelzyklen, Seil ohne PU-Beschichtung

Abbildung 8: Windentest mit 6mm-Technora-Seil

\subsection{Biegewechseltest}

Beim Lauf über Scheiben und Trommeln bilden sich dynamische Pressungen sowie Biege- und Zugspannungen in den Seillitzen aus, die zu einer Materialermüdung führen. Darüber hinaus verursacht der helixförmige Seilaufbau Relativbewegungen zwischen den Litzen, die einerseits eine reine materialseitige Beanspruchung verhindern, jedoch andererseits zu Verschleißbildung führen. Für eine sichere Anwendung von Faserseilen muss deren Haltbarkeit beim Lauf über Seilrollen, die sog. Biegewechselfestigkeit, der Auslegung des Seiltriebs zu Grunde gelegt werden.

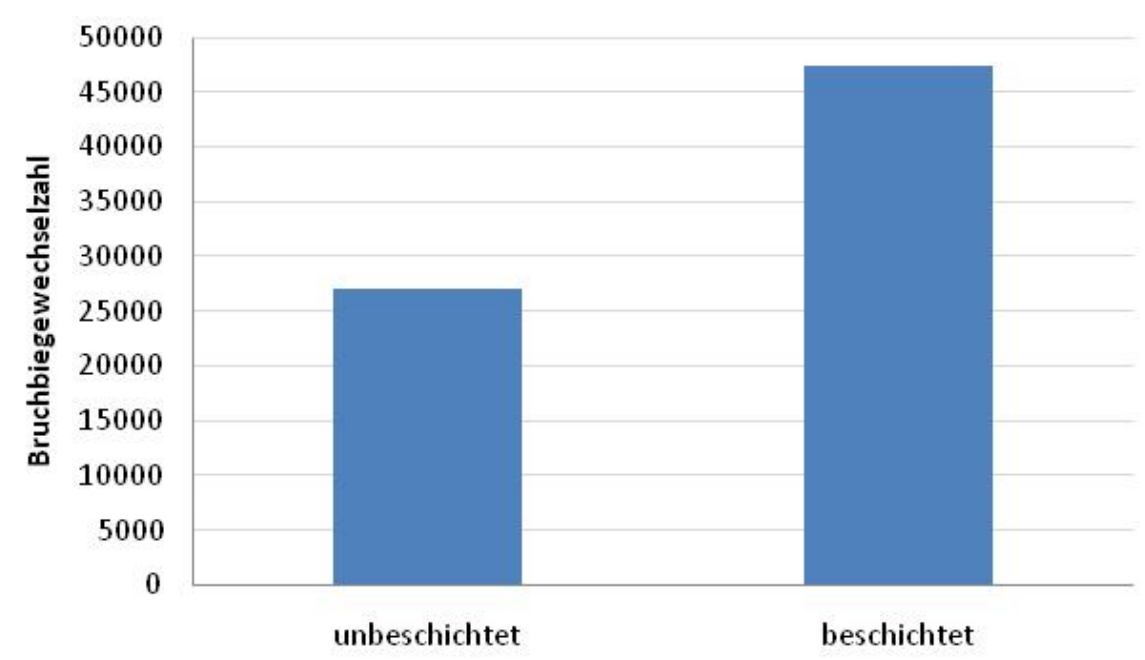

Abbildung 9: Bruchbiegewechselzahlen 
Erste vergleichende Untersuchungen mit imprägnierten Seilen wurden an einer Doppelbiegemaschine mit Standard-Rille, einer Prüfkraft von 25\% MBL und einem D/d-Verhältnis von 25 durchgeführt. Wie in Abbildung 9 zu sehen, zeigt sich, dass durch die Imprägnierung eine 175\%ige Erhöhung der Lebensdauer erreicht werden konnte. Dies ist vor allem mit der Behebung der eingangs beschriebenen Ursachen der Querschnitts-deformation und Faserknickung begründbar.

\subsection{Ausblick auf eine neue Tragmittelkonstruktion}

Die positiven Ergebnisse der Beschichtungsversuche motivieren eine neue Tragmittelkonstruktion, die im Folgenden kurz vorgestellt werden soll. Das Tragmittel besteht aus mehreren geflochtenen Zugseilen, die parallel angeordnet in eine elastische PU-Matrix eingebettet werden (Abbildung 10).

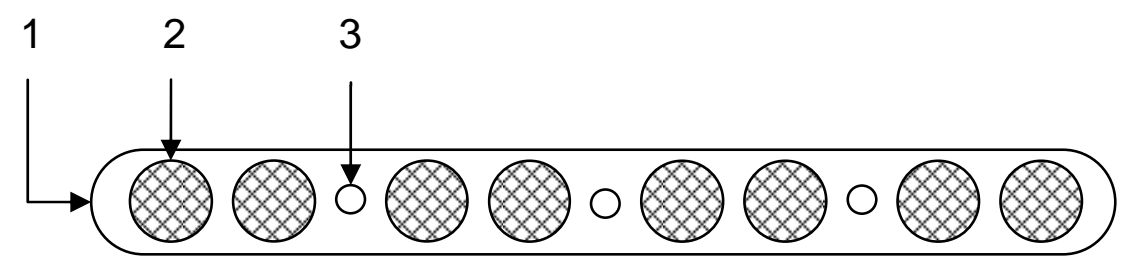

1 - Elastomeres Matrixmaterial

2 - Geflochtener Zugträger aus hochmodularen Polymerfasern mit Schmierstoff und ggf. Stützelement

3 - Wellenleiter

Abbildung 10: Prinzipieller Aufbau des Tragmittels

Der tragende Querschnitt teilt sich auf mehrere parallele Seilgeflechte auf. Dadurch wird die effektive Tragmittelhöhe verringert. Die Tragmittelkonstruktion ermöglicht so eine Vergrößerung des D/d-Verhältnisses (Scheibendurchmesser/Tragmittelhöhe) und mit ihm eine signifikante Erhöhung der Biegewechselfestigkeit.

Abbildung 11 zeigt ein CAD-Modell des neuartigen Tragmittels.
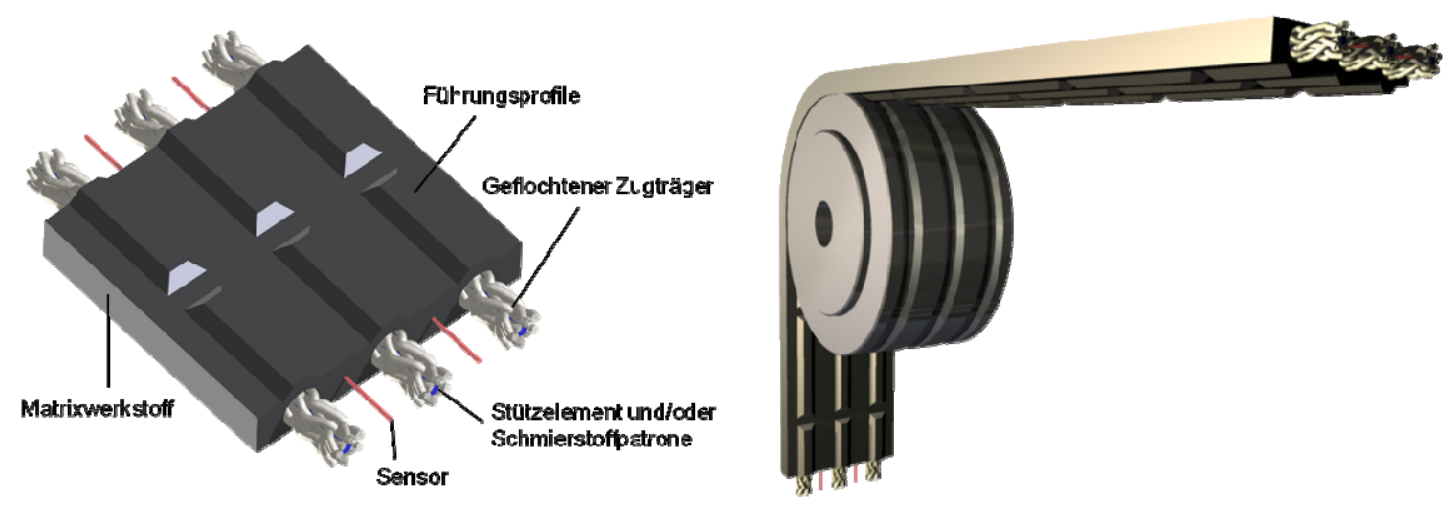

Abbildung 11: CAD-Modell des Tragmittels 
Die Seilgeflechte können gemeinsam mit parallel verlaufenden Wellenleitern (z.B. Lichtwellenleiter, elektrisch leitfähige Polymere), unter gleicher Vorspannung, in ein Elastomer eingebettet werden, wodurch Unordnungseffekte und ungleichmäßige Belastungen der Seilpartner und Wellenleiter ausgeschlossen werden können. Eingebettete Sensoren sind eine Möglichkeit den Zustand des Tragmittels zu überwachen. Durch den gegenüber den Zugträgern deutlich kleineren Durchmesser ist sichergestellt, dass die Sensoren beim Lauf über Scheiben nicht durch Querkräfte zerstört sondern nahezu vollständig auf Zug belastet werden.

Des Weiteren bewirkt der Matrixwerkstoff, wie bereits oben beschrieben, eine Reduzierung der lokalen Flächenpressungen, eine Verbesserung der Treibfähigkeit durch höhere Reibwerte und verhindert den Austritt von Schmiermittel aus dem Seilverband. Die maximal übertragbare Zugkraft ist bei herkömmlichen Riemen, mit Zugträgern aus Bündeln parallelliegender Fasern, begrenzt. Mit zunehmendem Strangdurchmesser werden dort die Zug- und Druckspannungen in der Biegezone unzulässig groß. Im Gegensatz dazu können durch die Verwendung der Seilgeflechte extreme Stranglasten, bei gleichzeitig guter Biegefestigkeit, übertragen werden.

Die labormäßige Herstellung des flachen Tragmittels (Abbildung 12) ist bislang in einer Gießtechnologie realisiert worden. Diese ermöglicht die Fertigung von ca. $2 \mathrm{~m}$ langen Mustern für erste Labortests.

Die Entwicklung einer Endlos-Fertigungstechnologie ist im Rahmen eines weiterführenden Projektes geplant.
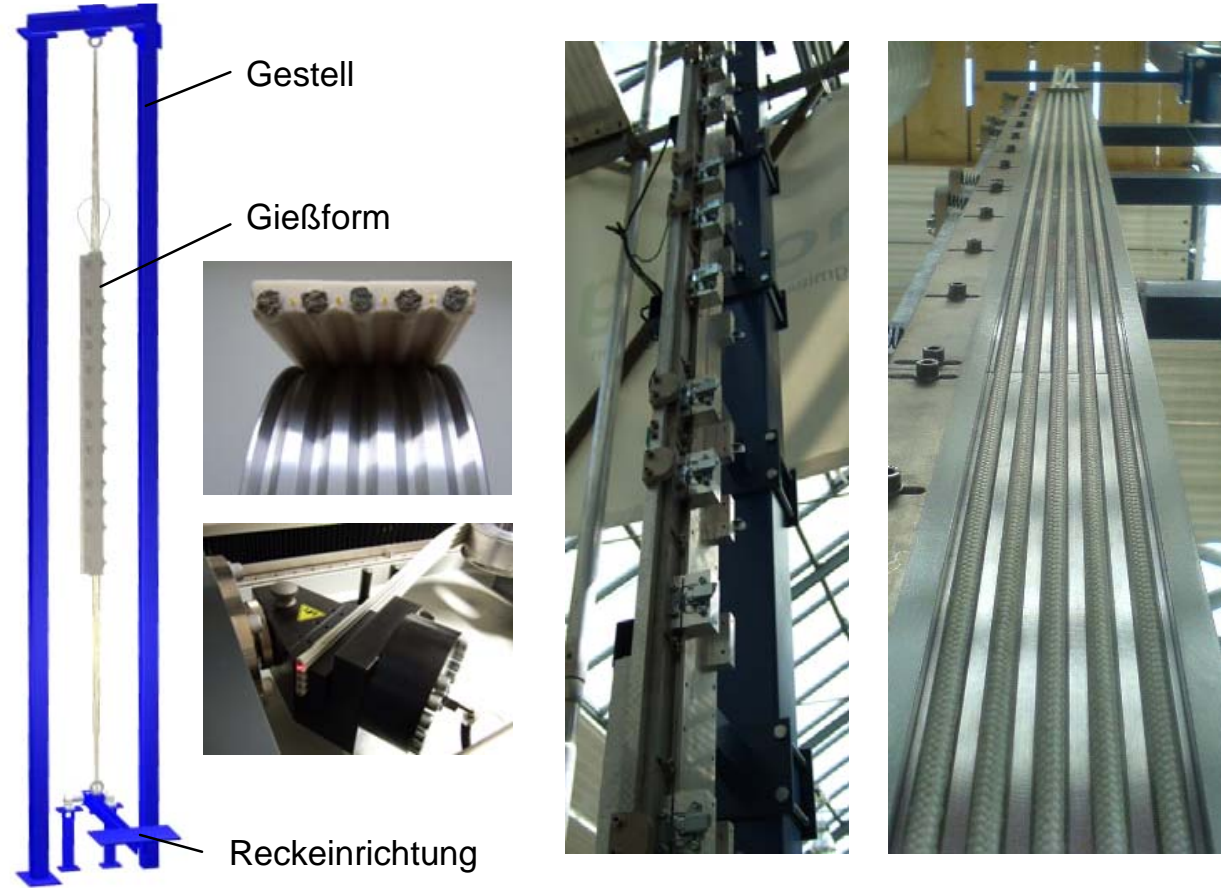

Abbildung 12: Gießvorrichtung 


\section{Literatur}

[Bla08] Blaasch, G.: Dubai Eldorado für Bauleute. Tiefbau Nr.4, 2008.

[Hea08] Hearle, J.W.S.: High performance fibres. CRC Press, 2008.

[Mor08] Morton, W.E.: Hearle, J.: Physical properties of textile fibres. Woodhead Publishing Limited, 2008.

[Oti10] Techreport Otis: Burji Khalifa, the world's tallest building inaugurated. Otis Elevator Company, 2010.

[Sie06] Techreport Siemag: Blair-Doppeltrommel-Fördermaschine für South Gold Mines. Siemag M-Tec, 2006.

[Ver05] Verreet, R.: Stahldrahtseile mit variablen Schlaglängen. Wire Rope Technology Aachen, 2005.

[Ver04] Verreet, R.: Eine kurze Geschichte des Drahtseiles. Wire Rope Technology Aachen, 2004.

[Vog98] Vogel, W.: Dauerbiegeversuche an gedrehten und geflochtenen Faserseilen aus hochfesten PE-Fasern. Technische Textilien 41, 1998. 